\title{
Segmentation of Liver from CT Abdominal Images
}

\author{
Hema N, M V Sudhamani
}

\begin{abstract}
Automatic segmentation of liver from the abdominal Computed Tomography images is a difficult task. It is very important to segment the liver accurately, so the tumors can be located, detected and classified accurately within a liver. The proposed segmentation methods include preprocessing stage as first step where image resizing and grayscale conversion is performed. Thresholding technique is applied to obtain a binary image. Next, liver is segmented from 2-D abdominal CT scanned images using various segmentation methods like adaptive thresholding with morphological operations, global thresholding with morphological operations and Watershed gradient transform. Next, Active contour balloon snake model is applied on 3-D dataset 3D-IRCADb (3D Image Reconstruction for Comparison of Algorithm Database). The empirical comparative study is carried out using JSC, DSC, sensitivity, specificity and accuracy and results are tabulated. The empirical comparative study of these methods using Dice and Jaccard Similarity Coefficient is carried out and results are tabulated.
\end{abstract}

Keywords - Abdominal Computed Tomography, Liver segmentation, Thresholding, Morphological operations, Watershed.

\section{INTRODUCTION}

Liver cancer is the fastest spreading cancer in India and worldwide. In India more than 11 lakh people undergo diagnosis for cancer every year. Nearly 6 lakh patients are dying in one year because of this disease. This is the second biggest reason of death in the country. Men are three times more liable than women to be diagnosed. Liver cancer is the fifth most and eighth most cause for death from cancer among men and women respectively.

Several techniques are used in medical imaging like, X-ray, Magnetic Resonance Image (MRI), Positron Emission Tomography (PET), CT scan and so on. In recent days, Computed Tomography (CT) scan is the frequently used imaging technique. X-ray technique is used to create the black and white pictures of inside the body. Different shades of images are created as the tissues absorb different quantity of the radiation. In CT scan imaging technique, sequence of $\mathrm{x}$-ray images taken at various angles are combined together and processing is carried out using computer system which helps to create cross sectional images of internal organs of the body. This technique is more specific when compared to a regular X-ray.

Revised Manuscript Received on December 12, 2019

* Correspondence Author

Hema N, Asst. Prof., ISE department, RNSIT, Bengaluru, India. Email: hemashekar05@gmail.com

Dr. M V Sudhamani, HoD, ISE department, RNSIT, Bengaluru, India. Email: mvsudha_raj@hotmail.com
CT scans shows tumor shape, size, and location accurately. By comparing CT scans done successively after the beginning of treatment, the doctors can analyze whether the tumor is responding to treatment or not.

The proposed work concentrates on accurately segmenting liver from the abdominal CT scanned image by ignoring other organs. The Abdominal CT scanned image is shown in below Figure 1.

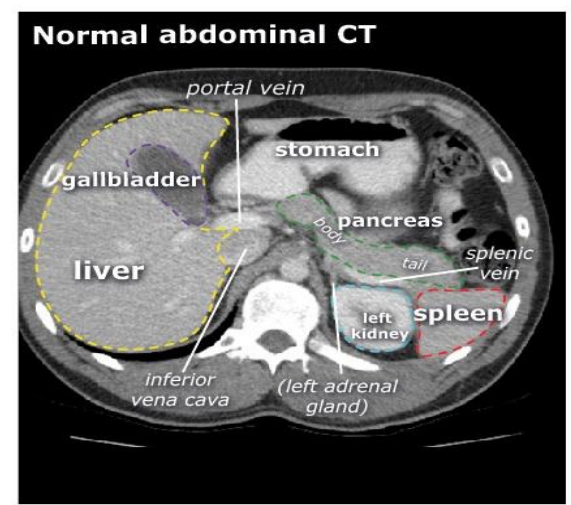

Figure 1 Abdominal CT scanned image

The process of dividing a digital image into many segments is known as segmentation. The main purpose of segmentation is to make changes to the view of an image into different form that will be easy to analyse $[6,11]$. Segmentation technique is used to find the objects and boundaries of our interest in images.

Various techniques have been implemented for automatic segmentation of liver. Some of the basic methods like thresholding, morphological operations and watershed transform are carried out and same has been discussed in this paper. The overview of the paper includes related work, methodology, results and discussions and conclusion.

\section{RELATED WORK}

Many Researchers have used various segmentation techniques to segment liver from the abdominal CT scanned Image.

In [1], an Edge Posterior Probability Model (EPPM) constrained adaptive image segmentation algorithm and improved watershed algorithm is implemented to segment the liver. Experiments have showed that the work has achieved good noise-free performance, accurate result, and it is useful in accurately segmenting low-contrast images. 
In [2], Watershed Transform segmentation method is applied to segment the liver. It completely divides images into separate areas even though the contrast is poor. The paragraph Watershed method has achieved $92.1 \%$ accuracy. In [3], the segmentation of liver is carried out using region growing method. Thresholding is applied by analyzing various intensity distributions and morphological erosion is applied on the image which reduces the computation time and efforts by removing other unwanted regions. This work has achieved $92.68 \%$ accuracy.

In [4], the liver is segmented by applying different methods such as, seeded region growing, label connected component algorithm and Neutrosophic set using thresholding. The author discusses the working of these methods and shows how these methods are used to segment the liver. Dice similarity measure is used to compare the segmentation result. The work has achieved $84 \%$ Dice similarity for Neutrosophic set with thresholding method. In [5], K-means clustering method is used to segment the liver image. Haar wavelet transform is used for feature extraction which finds the threshold values of the regions of interest. Finally, thresholding is applied to classify tumors as cancer or non-cancerous. The experiment has given an average accuracy of $82 \%$.

In [6], different image segmentation methods like edges based, region based, clustering based, watershed based and Artificial Neural Network Based are reviewed, discussed and assessment of their advantages and disadvantages are listed. From this it is understood that one sole method is not sufficient for all the images and all methods are not suitable for all the images. In [7], Watershed Transform is used to segment the liver. From the segmented liver image, the continuous and thin contours are identified and liver is accurately segmented. In [8], author discusses about the intensity analysis, region-growing and pre-processing techniques for automatically segmenting liver and tumor from abdominal CT scanned images. The Sensitivity of liver segmentation is $96.5 \pm 0.62 \%$ and Specificity is $99.2 \pm 0.6 \%$ respectively, and for tumor segmentation it is $92.6 \pm 0.33 \%$ and $99.8 \pm 0.1 \%$ respectively.

In [9], computer aided diagnostic system is designed for automatically extracting liver and tumor. The liver is segmented using region growing method. Thresholding technique is used for the analysis of intensity of the tumors and morphological erosion is applied to extract the tumors. In [10], Edge-based segmentation and region-based segmentation methods are integrated and as achieved more precise segmentation. The experimental outcome depicts that it is one of the strong algorithms and has obtained $93 \%$ accuracy in extracting the liver from abdominal CT.

In [11], comparative analysis of various liver image segmentation techniques such as Region based approaches, Threshold based approaches, Level Set Approach, Model based approach, and Active contour method and Gray Level method are discussed to illustrate the merits and demerits of various techniques that are available for segmentation. The main objective is to help in developing new segmentation techniques. In [12], Statistical shape model along with optimum surface detection strategy and watershed transform is used for liver segmentation and tumor is classified using Support Vector Machine. The method has achieved an overlap error of $29.49 \%$.

\section{METHODOLOGY}

The overall methodology in image processing as shown in Figure 2 consists of Input CT Image, Preprocessing, Liver Segmentation, Tumor Segmentation, Feature Detection and Extraction, and finally Tumor Classification. The liver is segmented from an 2-D abdominal CT scan images by means of Adaptive thresholding with Morphological operations, Global thresholding with Morphological operations and Gradient Watershed methods.

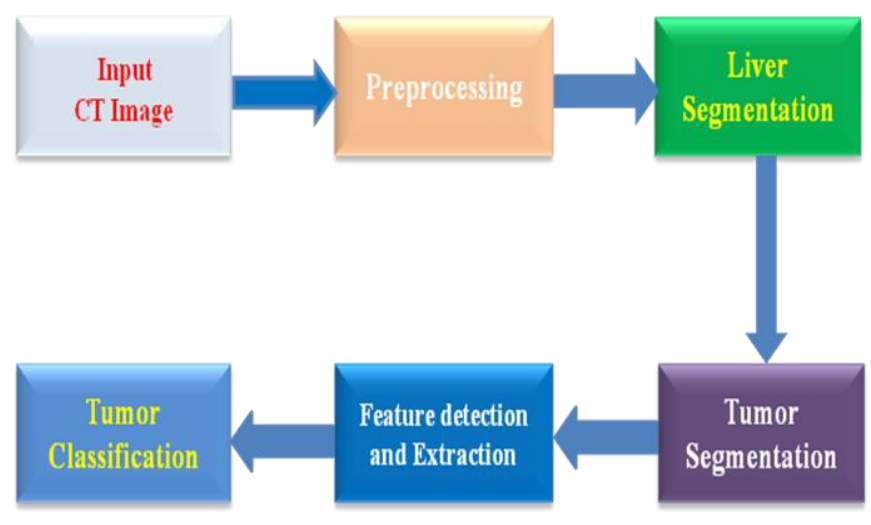

Figure 2 Proposed System Architecture

\section{A.Pre-processing}

The pre-processing step in the proposed work includes resizing an input image and converting the RGB images into gray scale images. The images considered are of various sizes. So, resizing an image is essential to convert all the input images into standard uniform size. Resizing changes, the dimension of an image so that it has the specified number of rows and columns. The input image is resized into 256 rows and 256 columns in the proposed work.

Next, the resized image has to be converted into gray scale image. RGB images are of 24-bits, in which each color components such as red, green and blue are of 8 bits. As this type of images yields millions of colors, in makes very difficult to process the images. So, conversion of RGB images into gray scale images is important as it gives less information about each pixel and speeds up further operations. In a resulted gray scale image each color component of an RGB image has equal intensity value when compared to three intensities in an RGB image. The intensities of gray scale images are stored using 8-bit integer which generates 256 different shades of gray from black to white i.e, 0-255.

Published By:

Blue Eyes Intelligence Engineering 


\section{B. Segmentation Methods}

The various algorithms used to segment the liver in the proposed work are discussed in detail.

\section{i. Adaptive Thresholding}

Thresholding is a simple technique which partitions an image into a foreground and background. Thresholding technique converts the gray scale images into binary images and separates the objects. The purpose of conversion of an image into binary image is important as the complexity of the data is reduced and simplifies the further recognition and classification process.

The simplest form of thresholding method replaces every pixel of an image with a black pixel when the intensity of an image is less than the threshold value $\mathrm{T}$ or replaces with white pixel when the intensity of image intensity is more than T. Adaptive thresholding technique [1] computes the threshold value locally. Mean of intensity of the $3 \times 3$ neighbourhood pixels are used as threshold value $\mathrm{T}$. Threshold value is calculated using below equation (1). The term $n$ represents the number of neighbourhood pixels and $a_{i}$ is the each pixel intensity value in the neighbourhood.

$$
\mathrm{T}=\frac{1}{\mathrm{n}} \sum_{\mathrm{a}=1}^{\mathrm{n}} \mathrm{a}_{\mathrm{i}}
$$

Morphological operations [3, 8] are applied on binary images. These operations need two inputs, one is original image and the other is the structuring element. In morphology technique, a structuring element is the shape or the shape of the image according to our requirement. It is consider as a matrix to identify every pixel of the image to be processed. It also defines about the neighbourhoods which are used in processing each pixel.

The basic operations of morphology are dilation and erosion. Greyscale dilation brightens small dark areas, and removes small dark holes. The dilation process is mathematically represented as below, where $\mathrm{y}$ is the set of reflectance of structuring element $\widehat{\mathrm{Y}}$ and the structuring

element $\mathrm{Y}$ is not equal to 0 .

$$
\mathrm{X} \oplus \mathrm{Y}=\{\mathrm{y} \mid \widehat{\mathrm{Y}} \cap \mathrm{X} \neq 0\}
$$

Greyscale erosion operation darkens bright areas, and minute bright areas like noise spikes will be removed. The erosion process is mathematically represented as below, where $\mathrm{y}$ is the set of points in an image $\mathrm{X}$, in which the structuring element $\mathrm{Y}$ is always within $\mathrm{A}$.

$$
\mathrm{X} \ominus \mathrm{Y}=\{\mathrm{y} \mid \mathrm{Y} \subseteq \mathrm{X}\}
$$

Dilation process adds some of the pixels to the boundaries of the objects in an image, where as erosion eliminates pixels present on boundaries of the object. The addition and elimination of the pixels from the object is based on size and shape of the structuring element used. a sub image. Structuring element is used to make changes in points in an image $\mathrm{X}$ such that the intersection between

The other morphological operations are opening and closing. Greyscale opening operation is carried out by eroding the image $\mathrm{X}$ with the chosen structuring element $\mathrm{Y}$, and then dilation is applied on the resulted image by using the structuring element. This process removes tiny bright spots like noises by darkening the small bright areas. The open operation is mathematically represented as below,

$$
\mathrm{X} \circ \mathrm{Y}=(\mathrm{X} \odot \mathrm{Y}) \oplus \mathrm{Y}
$$

Greyscale closing is carried out by dilating the image $\mathrm{X}$ using the structuring element $\mathrm{Y}$, and then erosion is applied on the resulted image by making use of the structuring element. This process brightens small dark areas and removes tiny dark holes. The close operation is mathematically represented as below. In the proposed work disk structuring element with a radius value 9 is used.

$$
\mathrm{X} \cdot \mathrm{Y}=(\mathrm{X} \oplus \mathrm{Y}) \Theta \mathrm{Y}
$$

Then morphological operations like open and erode are applied to the resulted binary image to separate the connected components. Then, the maximum connected component, liver is retrieved.

\section{Algorithm 1: Adaptive Thresholding}

Step 1: Pre-process the input image using median filter.

Step 2: Convert into binary image by applying adaptive thresholding method using equation (1).

Step 3: Perform morphological open using equation (4).

Step 4: Select the maximum connected component which is having maximum area.

\section{ii. Morphological Operations}

First, for the pre-processed image threshold value selected globally is applied to convert gray image into binary image. Global thresholding method [6] [11] uses same threshold value for all the pixels in the entire image. In this proposed work, threshold value 0.3 is set to obtain a binary image.

If the intensity value of an input image $f(x, y)$ is greater than the set threshold value 0.3 , then it will be replaced with intensity value 1 . If the intensity value is lesser than the set threshold value 0.3 , then it will be replaced with intensity value 0 . The resultant binary image obtained is $g(x, y)$. The mathematical representation is as shown below.

$$
\mathrm{g}(\mathrm{x}, \mathrm{y})= \begin{cases}1_{x} & \text { if } \mathrm{f}(\mathrm{x}, \mathrm{y})>T \\ 0, & \text { if } \mathrm{f}(\mathrm{x}, \mathrm{y}) \leq \mathrm{T}\end{cases}
$$

After obtaining binary image, morphological operations such as open and erode as discussed above are applied. Finally, a maximum area in the processed image, liver is extracted.

\section{Algorithm 2: Morphological Operations with Global} Thresholding 


\section{Segmentation of Liver from CT Abdominal Images}

Step 1: Pre-process the input image by applying median filter.

Step 2: Obtain binary image from gray scale image by applying global threshold method using equation (6).

Step 3: Apply morphological operations open and erode using equation (4) and (3) respectively.

Step 4: Locate maximally connected component.

\section{iii. Gradient Watershed}

Watershed segmentation method [2,7] is carried out on gray scale images. This method finds the watershed regions/ catchment basins and the ridge lines in a grey scale image. An input image is changed into a novel image with ridge lines and catchment basins. The objects that have to be segmented from an image are represented using catchment basins.

Watershed method estimates the label matrix which identifies the watershed regions in the input image. The elements of matrix are integers in whose values are more than or equal to 0 . The values of elements labelled 0 represents ridge lines and 1 represents the first watershed region and the values 2 represents the second watershed region, and so on.

Gradient magnitude image [12] is generated before applying watershed transform. The generated gradient magnitude image consists higher pixel values for object edges and small pixel values in remaining part of the object except edges. Gradient image is obtained by applying below equation where $g$ is the gradient image obtained, $f$ is the input gray scale image and $b$ is structuring element. The boundaries between regions and edges are enhanced in the gradient image $\mathrm{g}$.

$$
\mathrm{g}=(\mathrm{f} \oplus \mathrm{b})-(\mathrm{f} \Theta \mathrm{b})
$$

Watershed algorithm when carried on the gradient image results in over segmentation [7]. To avoid the over segmentation, markers concept is used. A connected component which belongs to an image is known as marker. Two form of markers exists such as, internal and external markers. Internal markers are related with the objects which have to be segmented and external markers associates with background of an image. Markers are obtained by applying operations such as reconstructed opening and closing which results in regional maxima and this act as a good foreground marker.

Two images are considered in carrying out the operations such as opening and closing by reconstruction. One is marker image $(F)$ and the other is mask image $(\mathrm{G})$. The connected component which belongs to an input image is a marker and mask is the input image. Opening by reconstruction $\mathrm{O}(\mathrm{F})$ is used to restore the original shapes of the objects that remain after erosion. The operation is mathematically represented as shown below, where $R_{D}$ is reconstruction by dilation and $B$ is the structuring element.

$$
\begin{gathered}
O(F)=R_{D}[F \ominus B] \\
R_{D}(F)=D_{G}(F)
\end{gathered}
$$

$\mathrm{D}_{\mathrm{G}}(\mathrm{F})$ is the geodesic dilation of $\mathrm{F}$ which is calculated using below equation.

$$
D_{G}(F)=(F \oplus B) \cap G
$$

Closing by reconstruction C(F) cleans up the opened image by eliminating small holes and fills the gaps that are present along with the boundary. The operation is mathematically represented as shown below, where $R_{E}$ represents reconstruction by erosion and B is a structuring element.

$$
\begin{array}{r}
C(F)=R_{E}[F \oplus B] \\
R_{E}(F)=E_{G}(F)
\end{array}
$$

$\mathrm{E}_{\mathrm{G}}(\mathrm{F})$ is the geodesic erosion of $\mathrm{F}$ which is calculated using below equation.

$$
E_{G}(F)=(F \ominus B) \cup G
$$

Once the maximum region is obtained it is superimposed on the input image to locate the liver image which is to be segmented.

\section{Algorithm 3: Gradient Watershed}

Step 1: Using median filter pre-process the input image .

Step 2: Obtain Gradient image.

Step 3: Apply watershed algorithm.

Step 4: Perform opening and closing by reconstruction.

Step 5: Obtain regional maxima.

Step 6: Superimpose regional maxima on the original image.

\section{iv. Liver Segmentation of 3-D Images using Active Contour Balloon Snake model}

Active contours are used in the domain of image processing to locate the contour of an object. Often the edge is not continuous, i.e. there might be holes along the edge, and spurious edges can be present because of noise. Active contours try to improve on this by imposing desirable properties such as continuity and smoothness to the contour of the object. This means that the active contour approach adds a certain degree of prior knowledge for dealing with the problem of finding the object contour.

An active contour is modeled as parametric curve, this curve aims to minimize its internal energy by moving into a local minimum. The position of the snake is given by the parametric curve $\mathrm{v}(\mathrm{s})=[\mathrm{x}(\mathrm{s})$, $y(s)]$. The below Figure 3 illustrates the parametric snake 
curve $v(s)$. The dot marks the starting point and end point of the snake curve.

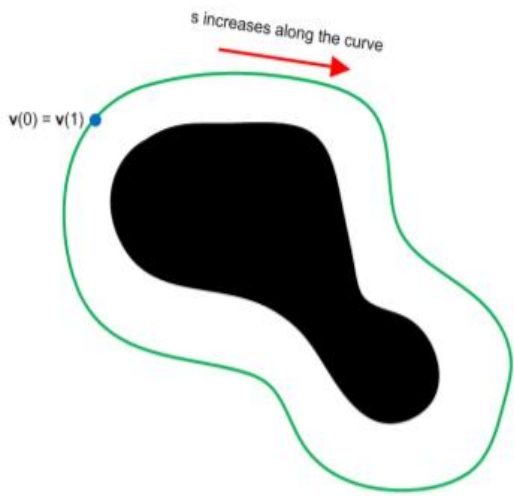

Figure 3 Illustration of a parametric snake curve

A snake is a parametric curve which tries to move into a position where its energy is minimized. The mathematical representations of energy minimization function for active contour snakes is given in general form as follows,

Contour is set of points,

$$
V(s)=\left(\left(x_{0}(s), y_{0}(s)\right),\left(x_{1}(s), y_{1}(s)\right), \ldots,\left(x_{n-1}(s), y_{n-1}(s)\right)\right)
$$

Energy function of curve or snake is given in below equation (15).

$$
\mathrm{E}_{\text {snake }}=\mathrm{E}_{\mathrm{in}}+\mathrm{E}_{\mathrm{ex}}
$$

Where $E_{\text {snake }}$ is total energy of contour and $E_{\text {in }}$ is internal energy of contour represents elasticity. External energy $E_{e x}$ of contour represents shape of the contour or stiffness. The detail and simplified formula for total energy function is given as follows,

$$
\begin{aligned}
E(V(s))= & \alpha \sum_{i=0}^{n-1}\left(\left(x_{i+1}(s)-\left(x_{i}(s)\right)^{2}+\left(y_{i+1}(s)-y_{i}(s)\right)^{2}-\sum_{i=0}^{n-1} \mid I_{x}\left(\left.\left(x_{i}(s), y_{i}(s)\right)\right|^{2}\right.\right.\right. \\
& +\mid I_{y}\left(x_{i}(s),\left.y_{j}(s)\right|^{2}\right.
\end{aligned}
$$

In the equation (16), $\alpha$ is relative weight factor and first term of summation corresponds to internal energy and second term is external energy of contour $V(s)$. The $I_{x}$ and $I_{y}$ are gradient image in the $\mathrm{x}$ and $\mathrm{y}$ directions respectively which are defined in the below equation and they corresponds to edge information of image. The objective of snakes is to find the minimum value of $\mathrm{E}_{\text {snake }}$ using gradient descent in order to iteratively locate final boundary of object present in an image.

The balloon snake model works on the same principles as the snake model, but where the snake would shrink under the influence of image forces but the balloon snake model expands. The expansion of the snake has some resemblance to a balloon thus this snake model is usually called the balloon snake. The expansive behavior is achieved by altering the values of $I_{x}$ and $I_{y}$ in the above equation.

The changes to the original snake is that the balloon snake makes it possible to find the contours of an object by placing the initial snake inside the object instead of outside, while also providing more stable results.

\section{Algorithm 4: Active Contour Balloon Snake model} Step 1: Preprocess the input image using median filter. Step 2: Balloon snake points are initialized first to generate the contour.

Step 3: Shrinking and expansion of a curve occurs until the balloon snake points reaches edges of an object.

Step 4: The region of interest is segmented and obtained as output image.

\section{RESULTS AND DISCUSSIONS}

The proposed methods are carried out to segment liver from CT scanned abdominal image. The input image is pre-processed first and then by applying the above discussed methods, the liver is segmented.

Output from various segmentation techniques applied on 2-D images are shown below.
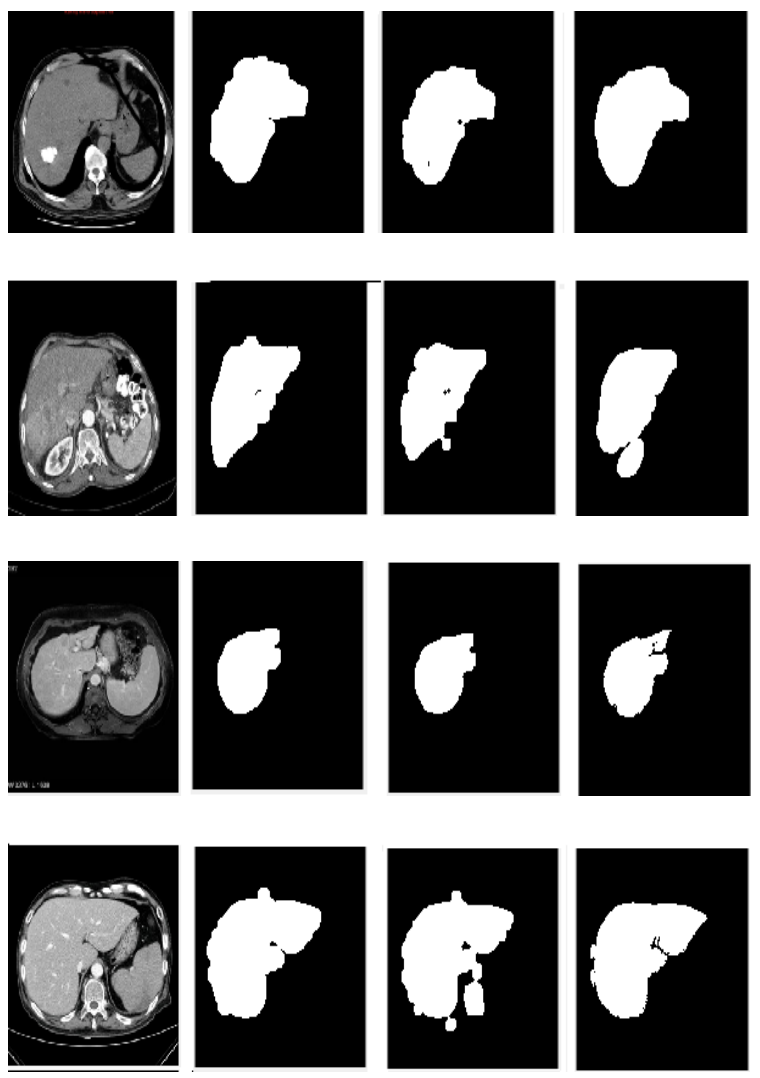

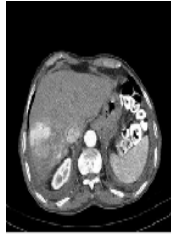

(a)

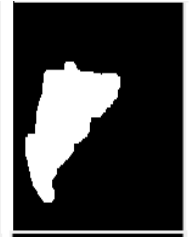

(b)

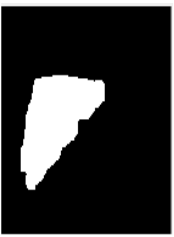

(c)

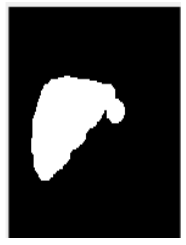

(d)

Figure 4 Results of Segmentation on 2-D images

a) Input Image b) Adaptive Thresholding with Morphological operations

c) Global thresholding with

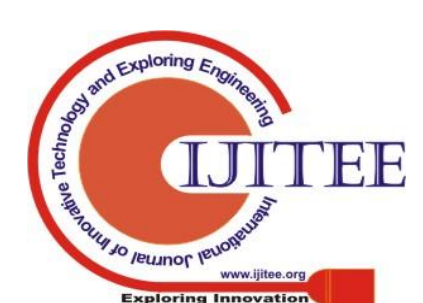




\section{Morphological Operations d) Gradient Watershed} segmentation

The comparative study [4] of segmentation techniques discussed above are carried out using Jaccard Similarity Coefficient (JSC) and Dice Similarity Coefficient (DSC).

Jaccard similarity is the ratio between the intersection and union of the segmented results $(\mathrm{X})$ and the ground truth result (manual segmentation result) (Y).

$$
\mathrm{I}(\mathrm{X}, \mathrm{Y})=\frac{|\mathrm{X} \cap \mathrm{Y}|}{|\mathrm{X} \cup \mathrm{Y}|}
$$

Dice score is 2 times the intersection between the segmentation results with the ground truth result (manual segmentation result) divided by the sum of both of them.

$$
\mathrm{D}(\mathrm{X}, \mathrm{Y})=\frac{2|\mathrm{X} \cap \mathrm{Y}|}{|\mathrm{X}|+|\mathrm{Y}|}
$$

The results are depicted in the table below. Morphological Transformation technique achieves better results when compared to other techniques.

Table 1 Empirical Comparative Study

\begin{tabular}{|c|c|c|}
\hline \multicolumn{1}{|c|}{ Table 1 Empirical Comparative Study } & DSC \\
\hline $\begin{array}{c}\text { Adaptive Thresholding with } \\
\text { Morphological operations }\end{array}$ & 89.19 & 94.21 \\
\hline $\begin{array}{c}\text { Global thresholding with } \\
\text { Morphological Operations }\end{array}$ & $\mathbf{9 1 . 9 5}$ & $\mathbf{9 5 . 7 3}$ \\
\hline Gradient Watershed & 85.56 & 92.13 \\
\hline
\end{tabular}

The above discussed Active Contour Balloon Snake model segmentation technique is applied on 3-D abdominal CT images and the outputs are shown below.
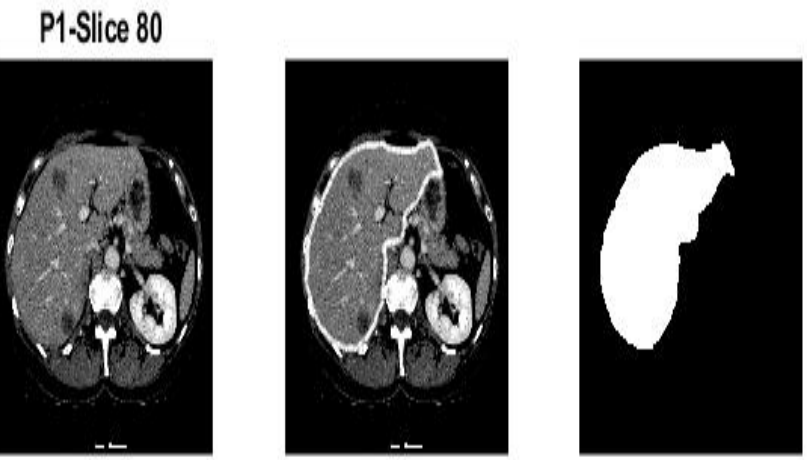

\section{P4-Slice 62}
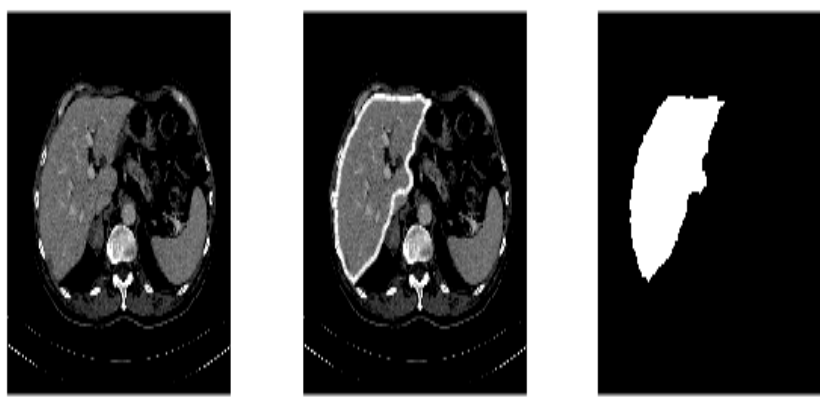

P8-Slice 88
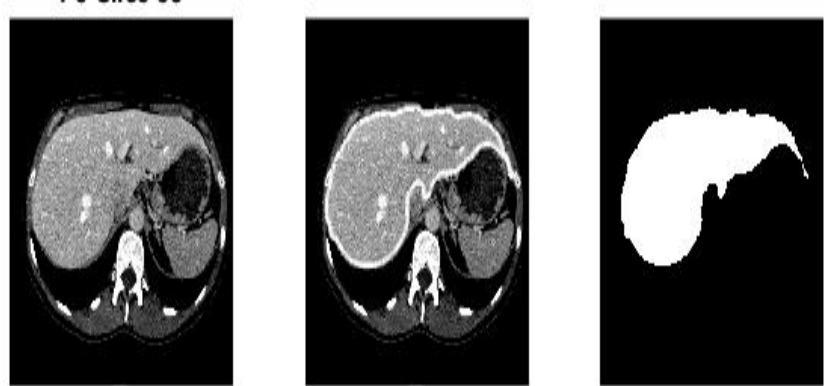

\section{Pg-Slice 73}
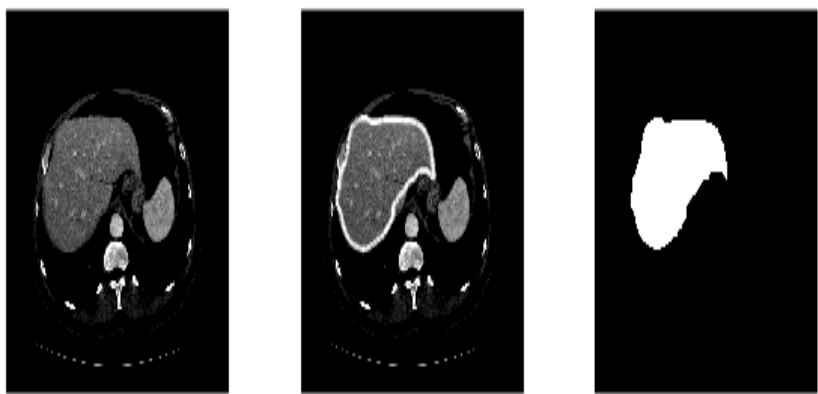

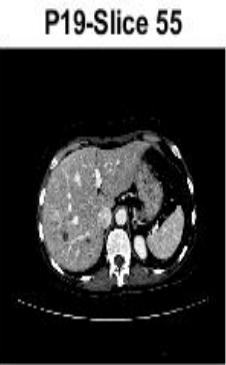

(a)

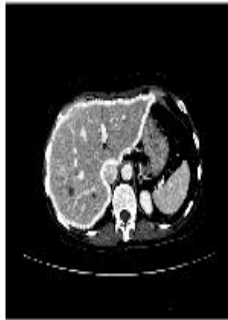

(b)

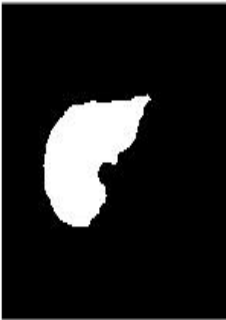

(c)
Figure 5 Results of Segmentation on 3-D images a) Input Image b) Output of Active Contour Balloon Snake model c) Segmented Liver

The number of pixels in the ground truth data (liver marked by the radiologist) and the number of pixels in the segmented liver is shown in below Table 2 . 
Table 2 Pixels Data

The Empirical Comparative Study of Active Contour Balloon Snake model has been done using various metrics like JSC, DSC, Sensitivity, Specificity and Accuracy. The values of five patients are shown in Table 3 .

Table 3 Empirical Comparative Study of Active Contour Balloon Snake model

\begin{tabular}{|c|c|c|c|c|c|c|c|}
\hline $\begin{array}{c}\text { Sl. } \\
\text { No. }\end{array}$ & $\begin{array}{c}\text { Patient } \\
\text { No. }\end{array}$ & Slice No. & $\begin{array}{c}\text { JSC } \\
(\%)\end{array}$ & DSC (\%) & $\begin{array}{c}\text { Sensitivity } \\
(\%)\end{array}$ & $\begin{array}{c}\text { Specificity } \\
(\%)\end{array}$ & $\begin{array}{c}\text { Accuracy } \\
(\%)\end{array}$ \\
\hline 1 & P1 & 80 & 95.21 & 97.5 & 98.97 & 99.18 & 99.08 \\
\hline 2 & P4 & 62 & 94.61 & 97.18 & 97.73 & 99.59 & 98.66 \\
\hline 3 & P8 & 88 & 95.66 & 97.73 & 98.1 & 99.45 & 98.78 \\
\hline 4 & P9 & 73 & 94.57 & 96.9 & 97.43 & 98.95 & 98.76 \\
\hline 5 & P19 & 55 & 95.05 & 97.38 & 98.78 & 99.52 & 99.15 \\
\hline & & Average & 95.02 & 97.33 & 98.2 & 99.33 & 98.88 \\
\hline
\end{tabular}

Table 4 Comparative study of proposed work with existing works

\begin{tabular}{|c|c|c|c|c|}
\hline $\begin{array}{l}\text { Sl. } \\
\text { No. }\end{array}$ & Author & $\begin{array}{l}\text { Segmenta } \\
\text { tion } \\
\text { Approach }\end{array}$ & $\begin{array}{l}\text { Data Set } \\
\text { and size }\end{array}$ & $\begin{array}{c}\text { Accurac } \\
\mathbf{y}\end{array}$ \\
\hline 1 & Nidaa et.al. & $\begin{array}{l}\text { Bayesian } \\
\text { Model }\end{array}$ & $\begin{array}{l}44 \text { clinical } \\
\text { cases } \\
\text { (2-D } \\
\text { images) }\end{array}$ & $87 \%$ \\
\hline 2 & $\begin{array}{l}\text { Abdalla } \\
\text { Mostafa et. } \\
\text { al. }\end{array}$ & $\begin{array}{l}\text { Artificial } \\
\text { Bee Colony } \\
\text { optimization }\end{array}$ & $\begin{array}{l}38 \text { clinical } \\
\text { cases } \\
\text { ( } 2-\mathrm{D} \\
\text { images) }\end{array}$ & $93.73 \%$ \\
\hline 3 & Avsar et.al. & $\begin{array}{l}\text { Watershed } \\
\text { algorithm }\end{array}$ & $\begin{array}{l}44 \text { clinical } \\
\text { cases } \\
\text { (2-D } \\
\text { images) }\end{array}$ & $95.64 \%$ \\
\hline 4 & $\begin{array}{l}\text { Lianfen } \\
\text { Huang et. } \\
\text { al. }\end{array}$ & $\begin{array}{l}\text { Single-block } \\
\text { Linear } \\
\text { Detection }\end{array}$ & $\begin{array}{l}\text { 3-DIRCA } \\
\text { DB } \\
20 \text { cases } \\
\text { (2-D } \\
\text { images) }\end{array}$ & $97.2 \%$ \\
\hline 5 & $\begin{array}{l}\text { Arvind H L, } \\
\text { Dr. M V } \\
\text { Sudhamani }\end{array}$ & $\begin{array}{l}\text { Top-hat } \\
\text { transform, } \\
\text { Adaptive } \\
\text { Region } \\
\text { growing }\end{array}$ & $\begin{array}{l}120 \text { cases } \\
\text { (2-D } \\
\text { images })\end{array}$ & $98.59 \%$ \\
\hline 6 & $\begin{array}{l}\text { Proposed } \\
\text { Method }\end{array}$ & $\begin{array}{l}\text { Active } \\
\text { Contour } \\
\text { Balloon } \\
\text { Snake } \\
\text { Model }\end{array}$ & $\begin{array}{l}3600 \\
\text { slices } \\
\text { (2-D } \\
\text { images) }\end{array}$ & $98.88 \%$ \\
\hline
\end{tabular}

\section{CONCLUSIONS}

This paper discussed about the segmentation of liver from 2-D abdominal CT using Adaptive thresholding with morphological operations, global thresholding along with morphological operations and Gradient Watershed methods. Empirical comparison has made between all these methods of segmentation and values obtained are tabulated. It was observed that global thresholding along with morphological operations technique performed better than other two. Next, Active Contour Balloon Snake model is applied on 3-D abdominal CT images to segment the liver. The average of

\begin{tabular}{|c|c|c|c|c|}
\hline $\begin{array}{l}\text { Sl. } \\
\text { No. }\end{array}$ & $\begin{array}{c}\text { Patient } \\
\text { No. }\end{array}$ & $\begin{array}{l}\text { Slice } \\
\text { No. }\end{array}$ & $\begin{array}{l}\text { No. of Pixels } \\
\text { in ground } \\
\text { truth liver }\end{array}$ & $\begin{array}{l}\text { No. of Pixels } \\
\text { in segmented } \\
\text { liver }\end{array}$ \\
\hline 1 & $\mathrm{P} 1$ & Slice 80 & 45859 & 47157 \\
\hline 2 & P4 & Slice 62 & 30199 & 30474 \\
\hline 3 & P8 & Slice 88 & 47990 & 48225 \\
\hline 4 & P9 & Slice 73 & 25856 & 25222 \\
\hline 5 & P19 & Slice 55 & 29100 & 29868 \\
\hline \multicolumn{5}{|c|}{$\begin{array}{l}\text { Jaccard and dice similarity coefficient, Sensitivity, } \\
\text { Specificity and Accuracy are used as evaluation parameter. } \\
\text { The values obtained for five different patients are tabulated. } \\
\text { In future, other advanced segmentation techniques have to be } \\
\text { explored. }\end{array}$} \\
\hline
\end{tabular}

\section{REFERENCES}

1. Meiling Gong1, Jinhui Lan1, Changlin Yang1, Hongtao Wu1, Tao Zhi1, "Adaptive image segmentation algorithm under the constraint of edge posterior probability", Institution of Engineering and Technology Comput. Vis., Vol. 11 Iss. 8, pp. 702-709, 2017.

2. Maithili Lawankar, Shraddha Sangewar and Somulu Gugulothu, "Segmentation of Liver using Marker Watershed Transform Algorithm for CT Scan Images", International Conference on Communication and Signal Processing, April 6-8, 2016.

3. Liaqat Ali, Khaled Khelil, "A Novel Fully Automated Liver and HCC Tumor Segmentation System Using Morphological Operations”, 2016.

4. M.Jayanthi, "Comparative Study of Different Techniques Used for medical image Segmentation of Liver from Abdominal CT Scan", WiSPNET 2016.

5. Anisha P R, Kishor Kumar Reddy C, Narasimha Prasad L V, "A pragmatic approach for detecting liver cancer using image processing and data mining techniques", Dept of ECE, K L University, SPACES-2015.

6. Dilpreet Kaur, Yadwinder Kaur, "Various Image Segmentation Techniques: A Review”, IJCSMC, Vol. 3, Issue. 5, pg.809 - 814, May 2014.

7. Amandeep Kaur, Aayushi, "Image Segmentation Using Watershed Transform", International Journal of Soft Computing and Engineering (IJSCE), ISSN: 2231-2307, Volume-4, Issue-1, March 2014.

8. O. Fekry Abd-Elaziz, M. Sharaf Sayed and M.Ibrahim Abdullah, Ola F.Abd-Elaziz, Mohammed S. Sayed and Mahmoud I. Abdullah, "Liver tumors segmentation from abdominal CT images using region growing and morphological processing", Pages: $1-6,2014$.

9. M. Jayanthi, Dr. B.Kanmani, "Extracting the liver and tumor from abdominal CT images", 5th International Conference on Signals and Image Processing, 2014.

10. Ahmed M. Anter, Ahmad Taher Azar Aboul Ella Hassanien, Nashwa El-Bendary, Mohamed Abu ElSoud, "Automatic computer aided segmentation for liver and hepatic lesions using hybrid segmentations technique", Proceedings of the Federated Conference on Computer Science and Information Systems, Pages: 193-198, 2013.

11. S.Priyadarsini, Dr. D.Selvathi, "Survey on Segmentation of Liver from CT Images", IEEE International Conference on Advanced Communication Control and Computing Technologies (ICACCCT), ISBN No. 978-1-4673-2048-1112, 2012.

12. Xing Zhang, Dehui Xiang, "Interactive Liver Tumor Segmentation from CT scans using Support Vector Classification with Watersheds", USA, IEEE, 2011. 


\section{AUTHORS PROFILE}

Mrs. Hema N, currently working as Assistant Professor, Dept. of ISE, RNSIT. She is having Teaching experience of 10 years and pursuing Ph.D in the area of Medical Image Processing.

Dr. M V Sudhamani, currently working as Dean-R\&D, Professor and HoD, Dept. of ISE, RNSIT. She is having Teaching, Research and Industrial experience of 25 years. She has specialization in Image Processing, Content-based Image Retrieval, Advanced Algorithms and Databases. Guided and guiding candidates for $\mathrm{Ph}$. D degree. She has carried out two research projects from VTU and AICTE. She has served as member of Board of Examiners (BOE) and Board of Study (BOS) member in VTU and other autonomous institutions across India. She has organized two international conferences ICDECS 2011 and 2015, and one more in December 2019. 